\title{
The Impact of Diabetes Mellitus and Hyperglycemia on the Severity and Outcome of Patients with COVID-19 Disease: A Single-Center Experience
}

\author{
Reem Al Argan (1) \\ Dania Alkhafaji' \\ Abdulmohsen Al Elq (D) \\ Waleed Albaker' \\ Safi Alqatari ${ }^{\prime}$ \\ Alaa Alzaki (D) \\ Abrar Alwaheed ${ }^{\prime}$ \\ Abir Al Said' \\ Huda Bukhari' \\ Sara Al Warthan' \\ Mohammad Zeeshan (iD) ${ }^{2}$ \\ Fatimah AIRubaish ${ }^{\prime}$ \\ Zainab AIElq' \\ Ahmed Alsahlawi \\ Mohannad Alalwan (ID) \\ Amani AlHwiesh' \\ Fatimah I Alabdrabalnabi ${ }^{1}$ \\ 'Department of Internal Medicine, \\ College of Medicine-Imam Abdulrahman \\ Bin Faisal University, King Fahd Hospital \\ of the University, Khobar, Eastern \\ Province, Saudi Arabia; ${ }^{2}$ Department of \\ Medical Education, College of Medicine, \\ Imam Abdulrahman Bin Faisal University, \\ King Fahd Hospital of the University, \\ Khobar, Eastern Province, Saudi Arabia
}

Correspondence: Reem Al Argan Department of Internal Medicine, College of Medicine, Imam Abdulrahman Bin Faisal University, King Fahd Hospital of the University, Khobar, Eastern Province, Saudi Arabia

Tel +966 I38957999 Extension 1842

Email Rjalarqan@iau.edu.sa
Purpose: Diabetes mellitus (DM) has been reported to be associated with a worse outcome of COVID-19 infection. The evidence is scarce in the Middle East and Saudi Arabia. We aimed to evaluate the impact of diabetes mellitus and hyperglycemia in non-diabetic individuals on the severity and outcome of COVID-19 infection.

Methods: This is a retrospective observational study, which included patients with confirmed COVID-19 infection [RT-PCR positive for SARS-CoV2] who were admitted to King Fahd Hospital of the University-Khobar-Eastern Province-Saudi Arabia from March to September 2020. Baseline demographic data, laboratory investigations, and markers of the severity of COVID-19 were analyzed. The collected data were categorized according to the Saudi Arabian Ministry of Health COVID-19 infection severity criteria. Patients were divided into three groups as follows: patients in Group 1 had pre-existing DM, patients in Group 2 did not have DM but were documented to have hyperglycemia at presentation, and patients in Group 3 were neither diabetics nor hyperglycemics at presentation and served as the control group. The severity and outcome of the control group were compared with the other two groups. The effect of risk factors on the severity and outcome of COVID-19 infection was studied in the DM group.

Results: A total of 414 patients were included (70.5\% males and $29.5 \%$ females). The mean age (SD) of patients was $52.3( \pm 15.5)$ years. Compared to the control group, pre-existing DM was found to be significantly associated with severe (OR 3.61), critical disease (OR 4.32), intensive care unit (ICU) admission (OR 2.0), and death (OR 2.0) from COVID-19 infection. Hyperglycemia without known DM was also found to be associated with critical COVID-19 pneumonia ( $\mathrm{P}$ 0.001), and had longer duration of hospitalization ( $\mathrm{P}$ 0.014), higher ICU admission, mechanical ventilation, and death from COVID-19 infection $(\mathrm{P}<0.0001)$.

Conclusion: Diabetes mellitus and hyperglycemia at presentation, even in the absence of preexisting DM, are independent risk factors for disease severity and worse outcome of COVID-19 infection. These patients should be identified and managed accordingly. The COVID-19 vaccination program should also target those populations to improve their outcomes.

Keywords: COVID-19 infection, diabetes mellitus, hyperglycemia, disease severity, disease outcome, mortality

\section{Introduction}

Since the start of the Corona virus disease-2019 (COVID-19) in Wuhan, China, the disease has spread rapidly to involve the whole world. It affected more than 264 million cases at the time of writing this paper. ${ }^{1}$ It has claimed more than 5 million lives worldwide, while new strains of the disease are still emerging. ${ }^{1}$ 
Saudi Arabia reported 548,474 COVID-19 cases to the World Health Organization with more than 8700 deaths as of October 29th, 2021. ${ }^{2}$ The spectrum of COVID-19 disease is highly variable. It ranges from mild disease in $80 \%$ of cases to critical disease in $5 \%$ of cases. ${ }^{3}$ Studies have shown that patients at high risk of severe COVID-19 infection or death have several characteristics, including advanced age, male sex, and have underlying health conditions, such as diabetes mellitus (DM), obesity and cardiovascular disease. ${ }^{4}$ Diabetes mellitus (DM) is recognised as a risk factor for several infections. ${ }^{5}$ Hyperglycaemia might support viral proliferation in COVID-19 infection via the production of mitochondrial reactive oxygen species and activation of hypoxia-inducible factor $1 \alpha^{6}$

Diabetes Mellitus was also found to be a common comorbidity with COVID-19. ${ }^{7,8}$ Previous reports have shown that $19-30 \%$ of COVID-19 patients have DM. ${ }^{7,8}$ Furthermore, a growing body of evidence supports the susceptibility to severe COVID-19 in diabetic patients. In a study of 500 patients from Wuhan city, a severe disease was found in $19 \%$ of diabetic patients. ${ }^{9}$ This was also illustrated in a study of 85 fatal cases where it was found that $22 \%$ of patients had DM. ${ }^{10}$ Severe pneumonia caused by COVID-19 infection predisposes diabetic patients to a higher need for critical care and mechanical ventilation. This was clearly demonstrated in an analysis carried out by Zhu et al, who studied more than 7000 patients from 19 hospitals in Hubei province, China. They found that having diabetes was associated with a higher rate of oxygen inhalation, non-invasive and invasive ventilation. ${ }^{11}$ Similar findings were reported in a meta-analysis of eight studies where patients with COVID-19 who have DM had an increased risk of intensive care unit (ICU) admission. ${ }^{12}$

The mortality rate of COVID-19 ranges between $1.3 \%$ and $2.7 \%{ }^{9,10,13,14}$ However, it could reach $15-49 \%$ in critical cases. ${ }^{4,15}$ In the same study by Zhu et al, the inhospital death rate during a 28-day follow-up period was significantly higher in patients with pre-existing Type 2 diabetes mellitus (T2DM) relative to non-diabetic individuals (7.8\% versus $2.7 \%$, P 0.001). ${ }^{10}$ After controlling for other confounders, the hazard ratio (HR) of all-cause mortality was 1.70 (95\% CI 1.29-2.24; P 0.001). ${ }^{11}$

Among the highly affected countries by DM, the Kingdom of Saudi Arabia (KSA) is one of the most highly affected ones. A recent meta-analysis showed that the prevalence of DM in Saudi Arabia is $32.8 \%$ and it is expected to increase exponentially. ${ }^{16}$ Therefore, the diabetic population constitutes a significant number of the
Saudi population who could be affected by COVID-19 infection. They should be recognised early and treated accordingly including proper control of blood glucose keeping in mind the effect of hyperglycemia on the severity and outcome of such infection for the reasons discussed above. As a result, improving their outcome. There is limited evidence in the Middle East and Gulf region about such a fatal pandemic. The main objective of this study is to evaluate the impact of diabetes mellitus and hyperglycemia in non-diabetic individuals on the severity and outcome of COVID-19 infection. We expect that the results of this study will add to the scarce evidence from this region regarding COVID-19 infection.

\section{Materials and Methods Data Collection}

The study was approved by the institutional review board of Imam Abdulrahman Bin Faisal University with an approval number of (IRB-2020-01-248). This is a retrospective observational study, which included patients with confirmed COVID-19 infection [RT-PCR positive for SARS-CoV2] who were admitted to King Fahd Hospital of the University-Khobar-Eastern ProvinceSaudi Arabia during the period of March 1, 2020, to September 30, 2020. Pregnant women and patients with malignancy, human immune deficiency virus, and immune deficiency syndromes were excluded due to the expected worst outcomes in such populations, ${ }^{17,18}$ so as to avoid their potential confounding effects. Four hundred and fourteen patients were included in the study.

For the whole population, the following data was collected: (1) Initial assessment [age, gender, nationality, and comorbidities]. (2) Laboratory investigations at their peak levels during hospitalization include: [complete blood count, renal profile with electrolytes, lactate dehydrogenase $(\mathrm{LDH})$, erythrocyte sedimentation rate (ESR), C-reactive protein (CRP), D-dimer and ferritin] (3) Data regarding severity of COVID-19 was collected based on patients' progress during hospitalization and was categorized based on the Saudi Arabian Ministry of health $(\mathrm{MOH})$ severity criteria ${ }^{19}$ as follows: A) Mild-moderate disease if there is no pneumonia on the chest $\mathrm{x}$-ray and there is no need for oxygen. B) Severe disease manifested by any of the following: respiratory rate $>30$ / minute, blood oxygen saturation $<93 \%$ on room air, partial pressure of oxygen/fraction of inspired oxygen $<300$, or lung infiltrates $>50 \%$ of the lung field within 
24-48 hours, C) Critical disease manifested by any of the following: adult respiratory distress syndrome (ARDS), sepsis, altered level of consciousness, multiorgan failure, or cytokine storm syndrome if there is ferritin $>600 \mathrm{ug} / \mathrm{L}$ at presentation and $\mathrm{LDH}>250$ or elevated D-Dimer $>1 \mathrm{mcg} / \mathrm{mL}^{19}$ The outcome of the COVID-19 disease was determined by the length of hospitalization, ICU admission, mechanical ventilation, and death of the patient.

Patients were divided into three groups: 1) Diabetes is defined as having a glycosylated hemoglobin (HBA1C) level of $6.5 \%$ or higher in the preceding three months. 2) Hyperglycemic group defined as having an HBA1C less than $6.5 \%$ and a random blood glucose (BG) level greater than $140 \mathrm{mg} / \mathrm{dl}$ at presentation without a history of diabetes. This BG cut off was chosen since it is compatible with the definition of inpatient hyperglycemia in many guidelines. $^{20,21}$ 3) Patients without DM or hyperglycemia, who will be referred to as the control group in this paper. Further data was collected regarding the DM group and included type of diabetes, laboratory and/or point of care glucose levels at presentation in addition to HBA1C.

Laboratory values, disease severity and outcome of COVID-19 infection were compared between the control and the other two groups. Further analysis had been carried out for the group with DM to study the effect of age, gender, type of diabetes, level of glucose and HBA1C on the severity and outcome of COVID-19 infection.

\section{Statistical Analysis}

Data were analyzed utilizing IBM SPSS.22. All categorical variables were presented as frequencies and percentages, while all continuous data was presented as Median and Interquartile range (IQR). Chi-square test or Fisher's exact test was used to check the association between variables. Kruskal Wallis test was used to compare the medians. Odds ratios (ORs) with their 95\% confidence intervals (CIs) were measured in a multivariate analysis. Statistical significance was set at $\mathrm{P}<0.05$.

\section{Results}

\section{Baseline Demographics and Comorbidities}

A total of 414 patients were included in this study. There were $70.5 \%$ males and $29.5 \%$ females. Majority (57.5\%) were Saudis. The mean age $( \pm \mathrm{SD})$ of patients was 52.3 $( \pm 15.5)$ years (range $18-93)$ years. Majority $47.6 \%$ were in the age group between 41 and 60 years followed by age 61-80 years and age 20-40 years represented by $25.6 \%$ and $23.2 \%$, respectively. $2.9 \%$ of patients were above the age of 80 years. The most prevalent comorbidities were hypertension $36.2 \%$, cardiac disease $13.3 \%$, chronic kidney disease (CKD) 8\%. Hematological and gastrointestinal (GI) diseases were present in $5.1 \%$ and $2.4 \%$, respectively. Only $1.7 \%$ had rheumatological diseases.

There were 197 cases (47.6\%) with DM, 36 cases $(8.7 \%)$ had hyperglycemia on presentation without preexisting DM, while 181 cases (43.7\%) were non-diabetic or hyperglycemic (control group). Out of 197 diabetic cases, 191 (97\%) had T2DM and only 6 (3\%) had type 1 diabetes mellitus (T1DM). The severity of COVID-19 disease for the total population was categorized based on Saudi Arabia MOH severity criteria. ${ }^{19}$ Out of 414 patients, 237 (57.2\%) were critical cases, 94 (22.7\%) were mildmoderate and $83(20 \%)$ were severe cases (Table 1).

\section{Laboratory Investigations}

Comparison of laboratory parameters between diabetic and control groups is presented in (Table 2). We found that white blood cell count (WBCs), neutrophils, blood urea nitrogen (BUN), creatinine, potassium (K), LDH, ESR, CRP and D-dimer levels were higher in the DM group $(\mathrm{P}<0.05)$. However, lymphocytes, sodium $(\mathrm{Na})$, chloride (Cl) and carbon dioxide (CO2) levels were lower in DM group ( $\mathrm{P}<0.05)$. Control group had higher ferritin level, but it was statistically insignificant ( $P$ 0.092). Hemoglobin and platelets counts were similar in both groups.

\section{Association Between DM and Severity of COVID-19 Infection}

Diabetes Mellitus was present in $51.5 \%$ of severe cases and $57.4 \%$ of critical cases $(\mathrm{P}<0.0001)$. The comparison of the severity of COVID-19 infection between diabetic and control groups have shown that out of 197 cases with DM, majority had critical disease (69.0\%) and severe disease $(21.8 \%)$ while only $9.1 \%$ presented with mildmoderate disease $(\mathrm{P}<0.0001)$. Significantly higher number of diabetic patients had ARDS $(\mathrm{P}<0.0001)$, sepsis $(\mathrm{P}$ $0.01)$, altered level of consciousness $(\mathrm{P}<0.001)$, multiorgan failure $(\mathrm{P}<0.002)$, cytokine storm syndrome with high ferritin and LDH (P 0.057) and high D-dimer $(\mathrm{P}<$ $0.0001)$. On the other hand, we found that a higher number of patients in the control group had mild-moderate disease criteria $(\mathrm{P}<0.0001)$ (Table 3$)$. 
Table I Baseline Characteristics

\begin{tabular}{|c|c|c|c|}
\hline & & Frequency & Percentage \\
\hline \multirow[t]{2}{*}{ Gender } & Male & 292 & 70.5 \\
\hline & Female & 122 & 29.5 \\
\hline \multirow[t]{2}{*}{ Nationality } & Saudi & 238 & 57.5 \\
\hline & Non Saudi & 176 & 42.5 \\
\hline \multirow{5}{*}{$\begin{array}{l}\text { Age Mean } \\
( \pm S D) 52.3 \\
( \pm 15.5) \text { years }\end{array}$} & $<20$ & 3 & 0.7 \\
\hline & $20-40$ & 96 & 23.2 \\
\hline & $4 I-60$ & 197 & 47.6 \\
\hline & $6 I-80$ & 106 & 25.6 \\
\hline & $>80$ & 12 & 2.9 \\
\hline \multirow[t]{7}{*}{ Comorbidities } & Hypertension & 150 & 36.2 \\
\hline & Chronic Kidney & 33 & 8 \\
\hline & Disease & & \\
\hline & Cardiac disease & 55 & 13.3 \\
\hline & $\begin{array}{l}\text { Hematologic } \\
\text { disease }\end{array}$ & 21 & 5.1 \\
\hline & $\begin{array}{l}\text { Gastrointestinal } \\
\text { disease }\end{array}$ & 10 & 2.4 \\
\hline & $\begin{array}{l}\text { Rheumatological } \\
\text { disease }\end{array}$ & 7 & 1.7 \\
\hline \multirow[t]{4}{*}{ Diabetes Status } & Diabetic & 197 & 47.6 \\
\hline & $\begin{array}{l}\text { Hyperglycemic } \\
\text { non-diabetic }\end{array}$ & 36 & 8.7 \\
\hline & Non-diabetic or & 181 & 43.7 \\
\hline & $\begin{array}{l}\text { Hyperglycemic } \\
\text { (Control) }\end{array}$ & & \\
\hline \multirow{2}{*}{$\begin{array}{l}\text { Type of } \\
\text { Diabetes }\end{array}$} & Type I & 6 & 3 \\
\hline & Type 2 & 191 & 97 \\
\hline \multirow{3}{*}{$\begin{array}{l}\text { Severity of } \\
\text { COVID-19 }\end{array}$} & Mild - Moderate & 94 & 22.7 \\
\hline & Severe & 83 & 20 \\
\hline & Critical & 237 & 57.2 \\
\hline
\end{tabular}

To study the effect of different risk factors on the severity of COVID-19 infection, a univariate analysis was carried out to evaluate the effect of age, gender, and co-morbidities (Table 4). Critical and severe diseases had significantly higher median age 56.5 and 52.0 years, respectively $(\mathrm{P}<$ $0.0001)$. Out of 237 critical cases, 62.3\% were males (P 0.004 ). In addition, $43.5 \%$ had hypertension (P 0.001) and $12.2 \%$ had CKD (P 0.001). Cardiac disease, hematological diseases, gastrointestinal (GI) diseases and rheumatological diseases had no effect on the severity. In multivariate analysis, DM was found to be an independent risk factor for both severe (OR 3.61) and critical (OR 4.32) COVID-19 infection. In addition, increasing age was found to be an independent risk factor for both severe (OR 1.04) and critical (OR 1.05) COVID-19 infection. Male gender and CKD were also both associated with critical diseases (OR 3.07 and 5.4), respectively (Table 4).

\section{Association Between DM and Outcome of COVID-19 Infection}

The outcome of COVID-19 infection was compared between diabetic and control group. We found that DM was significantly associated with longer duration of hospitalization, higher need for ICU admission, mechanical ventilation, and death rate $(\mathrm{P}<0.0001)$ (Table 5). Univariate analysis showed that DM was significantly associated with ICU admission OR 2.04 (95\% CI 1.33.1, P 0.001), mechanical ventilation OR 1.7 (95\% CI 1.1-2.7, P 0.017), and death OR 2.3 (95\% CI 1.3-4.0, P 0.004) (Table 6). On multivariate analysis, DM was found to be an independent risk factor for ICU admission OR 2.0 (95\% CI 1.3-3.1; P 0.045) and death OR 2.0 (95\% CI 1.1-3.7; P 0.04). Multivariate analysis also showed that age, male gender, GI, and rheumatologic diseases were independent risk factors for ICU admission. Old age, male gender and GI diseases were independent risk factors for mechanical ventilation. Old age and GI diseases were independent risk factors for death (Table 6).

\section{Association Between Hyperglycemia without Pre-Existing DM with Severity and Outcome of COVID-I 9 Infection}

We had 36 patients who presented with hyperglycemia without a known diagnosis of DM. We studied the association of hyperglycemia with the severity of COVID-19 infection, and we found that out of 36 cases, 75\% presented with critical COVID-19 pneumonia (P 0.001). Hyperglycemia was associated with ARDS, multi-organ failure and cytokine storm syndrome $(\mathrm{P}<0.05)$. In addition, we found that hyperglycemic group had longer duration of hospitalization (P 0.014), higher need for ICU admission, mechanical ventilation, and death $(\mathrm{P}<0.0001)$ (Table 7).

\section{Effect of Possible Risk Factors on the Severity and Outcome in Diabetic Group} Finally, we studied the effect of age, gender, type of diabetes, level of HBA1C and BG levels on the severity and outcome of COVID-19 infection. We found that male gender tended to have a higher risk of critical presentation (73.6\%, P 0.08) and older age $\geq 50$ years was associated with a higher requirement for mechanical ventilation (33.35\%, P 0.032) (Table 8). 
Table 2 Comparison of Laboratory Data Between Diabetic and Control Groups

\begin{tabular}{|c|c|c|c|c|c|}
\hline & Normal Range & $\begin{array}{l}\text { Total Cohort } \\
\text { Median (IQR) }\end{array}$ & $\begin{array}{l}\text { Diabetic Group } \\
\text { Median (IQR) }\end{array}$ & Control Group Median (IQR) & P-values \\
\hline WBC & $(4-\mathrm{II}) \mathrm{k} / \mathrm{ul}$ & $10(5.4-16.3)$ & $12(7-18)$ & $7.9(5-22)$ & $<0.001$ \\
\hline $\mathrm{Hgb}$ & $(12-16) \mathrm{g} / \mathrm{dl}$ & $13.2(|| .8-\mid 4.4)$ & $13(12-14)$ & $13.3(\mid 2.5-14.9)$ & 0.107 \\
\hline Platelets & $(140-450) \mathrm{k} / \mathrm{ul}$ & $213(158-267)$ & $212(152-264)$ & $214(161-269)$ & 0.413 \\
\hline Neutrophils & $(2-7.5) \mathrm{k} / \mathrm{ul}$ & $7.1(3.5-11.9)$ & $8.5(4.5-12)$ & $5.5(2.9-11.7)$ & 0.003 \\
\hline Lymphocytes & $(\mathrm{I}-5) \mathrm{k} / \mathrm{ul}$ & $1.2(0.8-2)$ & I.I5 (0.7-2) & $1.4(0.9-2)$ & 0.033 \\
\hline BUN & $(7-26) \mathrm{mg} / \mathrm{dl}$ & $13(10-19)$ & $16(11-22)$ & $12(9-15)$ & $<0.001$ \\
\hline Creatinine & $(0.6-1.3) \mathrm{mg} / \mathrm{dl}$ & $0.93(0.78-1.2)$ & I.04 (0.8-I.4) & $0.92(0.8-1.1)$ & $<0.001$ \\
\hline Sodium & $(136-146) \mathrm{mEq} / \mathrm{L}$ & $136(133-138)$ & $134(132-137)$ & $137(134-139)$ & $<0.001$ \\
\hline Potassium & $(3.5-5.1) \mathrm{mEq} / \mathrm{L}$ & $4.1(3.8-4.5)$ & $4.3(3.9-4.7)$ & $4(3.6-4.4)$ & $<0.001$ \\
\hline Chloride & $(98-107) \mathrm{mEq} / \mathrm{L}$ & $102(98-105)$ & $100(98-104)$ & $103(100-106)$ & $<0.001$ \\
\hline $\mathrm{CO} 2$ & $(20-31) \mathrm{mEq} / \mathrm{L}$ & $22(19.5-24)$ & $21(19-24)$ & $22(20-25)$ & 0.006 \\
\hline $\mathrm{LDH}$ & $(8 I-234) U / L$ & $434(303-652)$ & $455(338-704)$ & 402 (270-599) & 0.002 \\
\hline ESR & $(0-20) \mathrm{mm} /$ hour & $54(34-78)$ & $65(44-85)$ & $45(26-67)$ & $<0.001$ \\
\hline CRP & $(0.1-0.5) \mathrm{mg} / \mathrm{dl}$ & $10.6(4.5-18.4)$ & II.4 (6.4-19.6) & $9.6(2.6-17)$ & 0.004 \\
\hline D-dimer & $\leq 0.5 \mathrm{ug} / \mathrm{mL}$ & $1.08(0.505-2.6)$ & $1.35(0.66-3.35)$ & $0.77(0.43-I .4 I)$ & 0.001 \\
\hline Ferritin & $(21.8 \mathrm{I}-274.66) \mathrm{ng} / \mathrm{mL}$ & $582.1(245.5-1251)$ & $500(221-1097.8)$ & $619(273-1469)$ & 0.092 \\
\hline
\end{tabular}

Abbreviations: IQR, interquartile range; WBC, white blood cell; Hgb, hemoglobin; BUN, blood urea nitrogen; CO2, carbon dioxide; LDH, lactate dehydrogenase; ESR, erythrocyte sedimentation rate; CRP, C-reactive protein.

Table 3 Comparison of Severity of COVID-I9 Infection Between Diabetic and Control Group

\begin{tabular}{|c|c|c|c|c|}
\hline \multicolumn{2}{|c|}{ Severity of COVID-19 } & \multirow{2}{*}{$\begin{array}{c}\begin{array}{c}\text { Diabetic Group } \\
\text { Number }=197\end{array} \\
18(9.1 \%) \\
43(21.8 \%) \\
136(69 \%)\end{array}$} & \multirow{2}{*}{$\begin{array}{l}\begin{array}{l}\text { Non Diabetic or Hyperglycemic } \\
\text { (Control Group) Number = I I I }\end{array} \\
72(39.8 \%) \\
35(19.3 \%) \\
74(40.9 \%)\end{array}$} & \multirow{2}{*}{$\begin{array}{l}\text { P-value } \\
<0.0001\end{array}$} \\
\hline Severity & $\begin{array}{l}\text { Mild - Moderate } \\
\text { Severe } \\
\text { Critical }\end{array}$ & & & \\
\hline \multirow[t]{6}{*}{ Criteria } & \multicolumn{4}{|c|}{ Mild-Moderate } \\
\hline & $\begin{array}{l}\text { No pneumonia on chest x-ray } \\
\text { No } \mathrm{O} 2 \text { requirement }\end{array}$ & $\begin{array}{l}29(14.7 \%) \\
33(16.8 \%)\end{array}$ & $\begin{array}{l}70(38.7 \%) \\
80(44.2 \%)\end{array}$ & $\begin{array}{l}<0.0001 \\
<0.0001\end{array}$ \\
\hline & \multicolumn{4}{|c|}{ Severe } \\
\hline & $\begin{array}{l}\text { Respiratory rate }>30 / \mathrm{min} \\
\text { Oxygen saturation }<93 \% \text { on room air } \\
\mathrm{PaO} 2 / \mathrm{FiO} 2<300 \\
\text { Lung infiltrates }>50 \% \text { of lung field } \\
\text { within } 24-48 \text { hours }\end{array}$ & $\begin{aligned} 32 & (16.2 \%) \\
56 & (28.4 \%) \\
\text { I } & (0.5 \%) \\
4 I & (20.8 \%)\end{aligned}$ & $\begin{aligned} 27 & (14.9 \%) \\
46 & (25.4 \%) \\
1 & (0.6 \%) \\
28 & (15.5 \%)\end{aligned}$ & $\begin{array}{l}0.7 \\
0.5 \\
0.9 \\
0.2\end{array}$ \\
\hline & \multicolumn{4}{|c|}{ Critical } \\
\hline & $\begin{array}{l}\text { Adult respiratory distress syndrome } \\
\text { Sepsis } \\
\text { Altered level of consciousness } \\
\text { Multi organ failure } \\
\text { Cytokine Storm Syndrome: Ferritin }>600 \mathrm{ug} / \mathrm{L} \\
\text { at presentation and LDH }>250 \mathrm{U} / \mathrm{L} \\
\text { Cytokine Storm Syndrome: D-Dimer }>\text { I mcg/mL }\end{array}$ & $\begin{array}{l}62(31.5 \%) \\
18(9.1 \%) \\
21(10.7 \%) \\
13(6.6 \%) \\
63(32 \%) \\
79(40.1 \%)\end{array}$ & $\begin{aligned} 23 & (12.7 \%) \\
5 & (2.8 \%) \\
4 & (2.2 \%) \\
1 & (0.6 \%) \\
42 & (23.2 \%) \\
38 & (21 \%)\end{aligned}$ & $\begin{array}{c}<0.0001 \\
0.01 \\
0.001 \\
0.002 \\
0.057 \\
<0.0001\end{array}$ \\
\hline
\end{tabular}

Abbreviations: $\mathrm{PaO} 2$, partial pressure of oxygen; FiO2, fraction of inspired oxygen; $\mathrm{LDH}$, lactate dehydrogenase. 


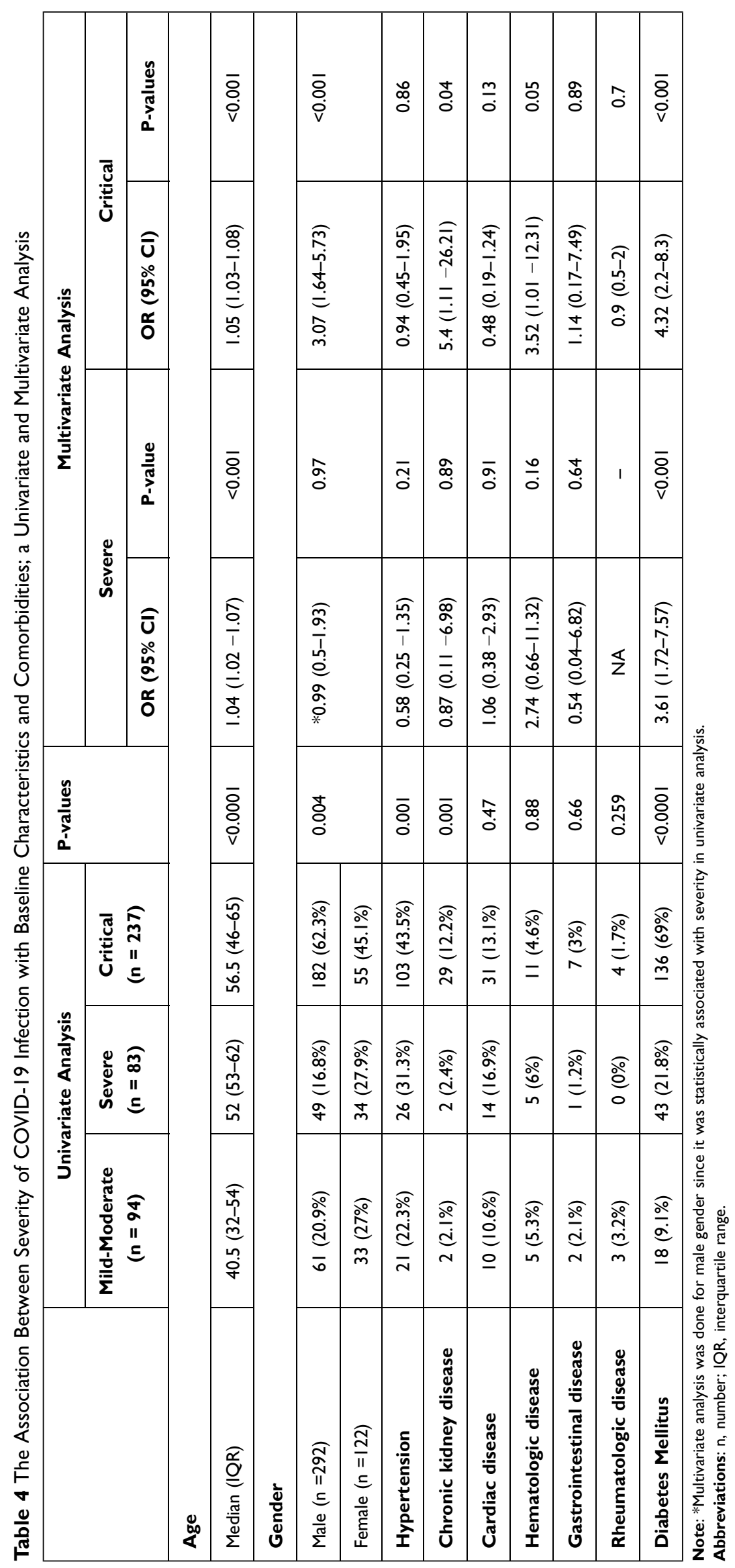


Table 5 Comparison of Outcomes Between Diabetic and Control Group

\begin{tabular}{|c|c|c|c|}
\hline Outcome Variables & $\begin{array}{l}\text { Diabetic Group Number } \\
=197\end{array}$ & $\begin{array}{c}\text { Non Diabetic or Hyperglycemic (Control Group) } \\
\text { Number }=181\end{array}$ & P-values \\
\hline $\begin{array}{l}\text { Duration of hospitalization Mean } \\
\pm \mathrm{SD} \text { (days) }\end{array}$ & $14.3 \pm 12.7$ & $10.2 \pm 9.5$ & $<0.0001$ \\
\hline ICU Admission & $84(42.6 \%)$ & 39 (21.5\%) & $<0.0001$ \\
\hline Need for ventilation & $58(29.4 \%)$ & $26(14.4 \%)$ & $<0.0001$ \\
\hline Death & $40(20.3 \%)$ & $10(5.5 \%)$ & $<0.0001$ \\
\hline
\end{tabular}

\section{Discussion}

In this study of 414 patients with COVID-19 infection, we have found the following key findings: To begin, the mean age of our population was 52.3 years, $70 \%$ were males, $47.6 \%$ had DM, and the majority had severecritical COVID-19 disease. Second, diabetic patients had higher inflammatory markers, worse kidney function, and lower $\mathrm{CO} 2$ levels. Next, most diabetic patients presented with severe-critical COVID-19 disease, had worse outcomes in terms of longer hospitalization, higher need for ICU admission, mechanical ventilation, and death. Moreover, multivariate analysis showed that DM was an independent risk factor for severe-critical COVID-19 disease, ICU admission and death. Interestingly, hyperglycemia without pre-existing DM was associated with critical COVID-19 pneumonia and worse outcome. In the DM

Table 6 Association Between Outcome Variables of COVID-19 Infection with Baseline Characteristics and Comorbidities; a Univariate and Multivariate Analysis

\begin{tabular}{|c|c|c|c|c|c|c|}
\hline \multirow[t]{2}{*}{ Variable } & \multicolumn{3}{|c|}{ Univariate Analysis } & \multicolumn{3}{|c|}{ Multivariate Analysis } \\
\hline & ICU Admission & $\begin{array}{l}\text { Mechanical } \\
\text { Ventilation }\end{array}$ & Death & ICU Admission & $\begin{array}{l}\text { Mechanical } \\
\text { Ventilation }\end{array}$ & Death \\
\hline Age & $\begin{array}{c}1.01(0.94-1) \\
P=0.00 I\end{array}$ & $\begin{array}{c}1.01(0.94-1) \\
P=0.00 I\end{array}$ & $\begin{array}{c}1.01(0.94-1) \\
P=0.00 I\end{array}$ & $\begin{array}{c}1.04(1.02-1.05) \\
\mathbf{P}<0.001\end{array}$ & $\begin{array}{c}1.03(1.01-1.05) \\
\mathbf{P}<0.001\end{array}$ & $\begin{array}{c}\text { I.I }(1.03-1.08) \\
P<0.00 \text { I }\end{array}$ \\
\hline Males & $\begin{array}{c}\mathrm{I} .5(0.96-2.4) \\
\mathrm{P}=0.08\end{array}$ & $\begin{array}{c}1.5(0.9-2.6) \\
P=0.13\end{array}$ & $\begin{array}{c}1.5(0.8-2.9) \\
\quad P=0.2\end{array}$ & $\begin{array}{c}1.9(1.1-3.1) \\
P=0.015\end{array}$ & $\begin{array}{c}1.9(1 . I-3.4) \\
P=0.03\end{array}$ & $\begin{array}{l}2(I-4) \\
P=0.06\end{array}$ \\
\hline Hypertension & $\begin{array}{c}1.7(1.1-2.6) \\
P=0.013\end{array}$ & $\begin{array}{l}1.9(1.2-3) \\
P=0.005\end{array}$ & $\begin{array}{c}1.8(1.1-3.1) \\
\quad P=0.3\end{array}$ & $\begin{array}{c}1.5(0.9-2.4) \\
P=0.9\end{array}$ & $\begin{array}{c}1.6(0.9-2.8) \\
P=0.6\end{array}$ & $\begin{array}{c}\mathrm{I} .5(0.8-2.8) \\
\mathrm{P}=0.7\end{array}$ \\
\hline $\begin{array}{l}\text { Chronic kidney } \\
\text { disease }\end{array}$ & $\begin{array}{c}1.3(0.6-2.6) \\
P=0.5\end{array}$ & $\begin{array}{c}1.6(0.8-3.6) \\
P=0.2\end{array}$ & $\begin{array}{c}1.6(0.7-3.9) \\
P=0.2\end{array}$ & $\begin{array}{c}0.8(0.4-1.9) \\
\quad P=0.6\end{array}$ & $\begin{array}{l}\text { I.I }(0.5-2.6) \\
\quad P=0.8\end{array}$ & $\begin{array}{l}\mathrm{I}(0.4-2.8) \\
\mathrm{P}=0.99\end{array}$ \\
\hline Cardiac disease & $\begin{array}{c}1.2(0.7-2.2) \\
\quad P=0.5\end{array}$ & $\begin{array}{c}\text { I.6 }(0.9-3) \\
P=0.1\end{array}$ & $\begin{array}{c}1.7(0.9-3.7) \\
\quad P=0.2\end{array}$ & $\begin{array}{c}0.8(0.4-1.6) \\
\quad P=0.2\end{array}$ & $\begin{array}{c}1.2(0.6-2.3) \\
P=0.9\end{array}$ & $\begin{array}{c}\text { I.3 }(0.6-3) \\
P=0.9\end{array}$ \\
\hline $\begin{array}{l}\text { Hematologic } \\
\text { disease }\end{array}$ & $\begin{array}{c}0.19(0.04-0.8) \\
P=0.014\end{array}$ & $\begin{array}{c}0.15(0.02-1.1) \\
P=0.3\end{array}$ & $\begin{array}{c}0.3(0.04-2) \\
\quad P=0.2\end{array}$ & $\begin{array}{c}0.2(0-0.9) \\
P=0.09\end{array}$ & $\begin{array}{c}0.2(0-1.3) \\
P=0.2\end{array}$ & $\begin{array}{c}0.4(0-3.1) \\
P=0.6\end{array}$ \\
\hline $\begin{array}{l}\text { Gastrointestinal } \\
\text { disease }\end{array}$ & $\begin{array}{c}4.6(I . I-18.3) \\
P=0.016\end{array}$ & $\begin{array}{c}3.2(0.9-1 \mid .4) \\
P=0.053\end{array}$ & $\begin{array}{c}6.1(1.7-2 \mid .7) \\
P=0.002\end{array}$ & $\begin{array}{c}7.2(|.7-3| . \mid) \\
\mathbf{P}=0.013\end{array}$ & $\begin{array}{c}4.6(1.2-17.8) \\
P=0.045\end{array}$ & $\begin{array}{c}8.6(2.2-33.6) \\
P=0.005\end{array}$ \\
\hline $\begin{array}{l}\text { Rheumatological } \\
\text { disease }\end{array}$ & $\begin{array}{c}2.6(0.6-\mid I .7) \\
P=0.2\end{array}$ & $\begin{array}{c}2.4(0.5-1 \mathrm{I}) \\
\quad P=0.2\end{array}$ & NA & $\begin{array}{c}5.9(1-35) \\
P=0.028\end{array}$ & $\begin{array}{c}\text { 4.I }(0.7-23.3) \\
\quad P=0.08\end{array}$ & NA \\
\hline Diabetes Mellitus & $\begin{array}{c}2.04(1.3-3.1) \\
P=0.00 I\end{array}$ & $\begin{array}{c}1.7(1.1-2.7) \\
P=0.017\end{array}$ & $\begin{array}{c}2.3(1.3-4) \\
P=0.004\end{array}$ & $\begin{array}{c}2(1.3-3.1) \\
P=0.045\end{array}$ & $\begin{array}{c}1.5(0.9-2.5) \\
P=0.39\end{array}$ & $\begin{array}{c}2(1.1-3.7) \\
P=0.04\end{array}$ \\
\hline
\end{tabular}

Note: Significant $P$ values are shown in bold.

Abbreviation: NA, not applicable. 
Table 7 Comparison of Severity and Outcome of COVID-19 Disease Between Hyperglycemic and Control Group

\begin{tabular}{|c|c|c|c|c|}
\hline & Variable & $\begin{array}{c}\text { Hyperglycemic Non Diabetic } \\
\text { Group Number }=36\end{array}$ & $\begin{array}{l}\text { Control Group } \\
\text { Number }=|8|\end{array}$ & P-values \\
\hline Severity & $\begin{array}{l}\text { Mild-Moderate } \\
\text { Severe } \\
\text { Critical }\end{array}$ & $\begin{array}{l}4(11.1 \%) \\
5(13.9 \%) \\
27(75 \%)\end{array}$ & $\begin{array}{l}72(39.8 \%) \\
35(19.3 \%) \\
74(40.9 \%)\end{array}$ & 0.001 \\
\hline \multirow{6}{*}{$\begin{array}{l}\text { Severity } \\
\text { Criteria }\end{array}$} & \multicolumn{4}{|c|}{ Mild-Moderate } \\
\hline & $\begin{array}{l}\text { No pneumonia on chest } x \text {-ray } \\
\text { No Oxygen requirement }\end{array}$ & $\begin{array}{l}5(13.9 \%) \\
6(16.7 \%)\end{array}$ & $\begin{array}{l}70(38.7 \%) \\
80(44.2 \%)\end{array}$ & $\begin{array}{l}0.004 \\
0.002\end{array}$ \\
\hline & \multicolumn{4}{|c|}{ Severe } \\
\hline & $\begin{array}{l}\text { Respiratory rate }>30 / \text { minute } \\
\text { Oxygen saturation }<93 \% \text { on room air } \\
\text { Pao2/Fio } 2<300 \\
\text { Lung infiltrates }>50 \% \text { of lung field within } 24-48 \\
\text { hours }\end{array}$ & $\begin{array}{c}6(16.7 \%) \\
6(16.7 \%) \\
0(0 \%) \\
4(11.1 \%)\end{array}$ & $\begin{aligned} 27 & (14.9 \%) \\
46 & (25.4 \%) \\
1 & (0.6 \%) \\
28 & (15.5 \%)\end{aligned}$ & $\begin{array}{l}0.8 \\
0.2 \\
0.7 \\
0.5\end{array}$ \\
\hline & \multicolumn{4}{|c|}{ Critical } \\
\hline & $\begin{array}{l}\text { Adult respiratory distress syndrome } \\
\text { Sepsis } \\
\text { Altered level of consciousness } \\
\text { Multi organ failure } \\
\text { Cytokine Storm: Ferritin }>600 \mathrm{ug} / \mathrm{L} \text { at } \\
\text { presentation and } \mathrm{LDH}>250 \mathrm{U} / \mathrm{L} \\
\text { Cytokine Storm: D-Dimer }>\text { I mcg/mL }\end{array}$ & $\begin{aligned} 13 & (36.1 \%) \\
2 & (5.6 \%) \\
1 & (2.8 \%) \\
3 & (8.3 \%) \\
20 & (55.6 \%) \\
17 & (47.2 \%)\end{aligned}$ & $\begin{aligned} 23 & (12.7 \%) \\
5 & (2.8 \%) \\
4 & (2.2 \%) \\
1 & (0.6 \%) \\
42 & (23.2 \%) \\
38 & (21 \%)\end{aligned}$ & $\begin{array}{c}0.001 \\
0.4 \\
0.8 \\
0.002 \\
<0.0001 \\
0.001\end{array}$ \\
\hline Outcome & $\begin{array}{l}\text { Hospital Stay Mean } \pm \text { SD (days) } \\
\text { ICU Admission } \\
\text { Mechanical ventilation } \\
\text { Death }\end{array}$ & $\begin{array}{l}16.3 \pm 13.7 \\
19(52.8 \%) \\
16(44.4 \%) \\
12(33.3 \%)\end{array}$ & $\begin{array}{c}10.2 \pm 9.5 \\
39(21.5 \%) \\
26(14.4 \%) \\
10(5.5 \%)\end{array}$ & $\begin{array}{c}0.014 \\
<0.0001 \\
<0.0001 \\
<0.0001\end{array}$ \\
\hline
\end{tabular}

Abbreviations: $\mathrm{PaO} 2$, partial pressure of oxygen; FiO2, fraction of inspired oxygen; $\mathrm{LDH}$, lactate dehydrogenase; ICU, intensive care unit.

group, male gender tended to have critical COVID-19 disease, and age $\geq 50$ years was associated with a higher risk of mechanical ventilation. Among other risk factors, age was remarkably associated with severe-critical COVID-19 disease, worse outcome, and death. Male gender was associated with critical disease, ICU admission, and mechanical ventilation. CKD was associated only with critical presentation. GI diseases were associated with worse outcome.

A recent paper by Alguwaihes et al from Saudi Arabia reported similar results to our findings with a median age of 55 years, male gender in $68 \%$ of cases with DM in $68 \%$ of COVID-19 cases. ${ }^{22}$ Similarly, almost half of our cohort had either DM or hyperglycemia. Bode et al studied more than 1000 COVID-19 patients from 88 United States hospitals and found that $38.5 \%$ were having either diabetes by
A1C criteria or uncontrolled hyperglycemia. ${ }^{23}$ However, data from other countries reported a much lower prevalence of $10-17 \%{ }^{24,25}$ The higher prevalence in our study and Alguwaihes et al paper can be explained by the high prevalence of DM in KSA. This was reported in a recent meta-analysis where prevalence of DM in KSA was expected to reach $35.37 \%$ in 2020 and $45.36 \%$ in the year $2030 .^{16}$

Most of our cohort presented with severe and critical COVID-19 pneumonia. This is in contrast with the expected severity pattern that most COVID-19 patients present with mild-moderate disease and a minority presents with severe-critical disease. ${ }^{3}$ This can be explained by the fact that our cohort is a hospital based rather than a community based where mainly patients with severecritical disease are being admitted. These findings are close to the data published by Wang et al who studied 


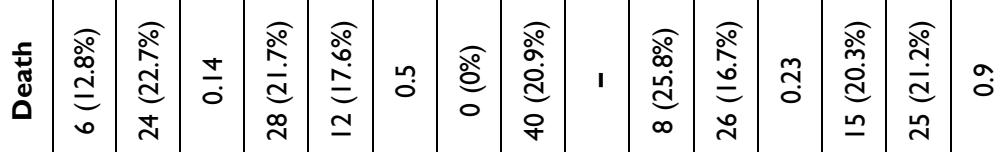

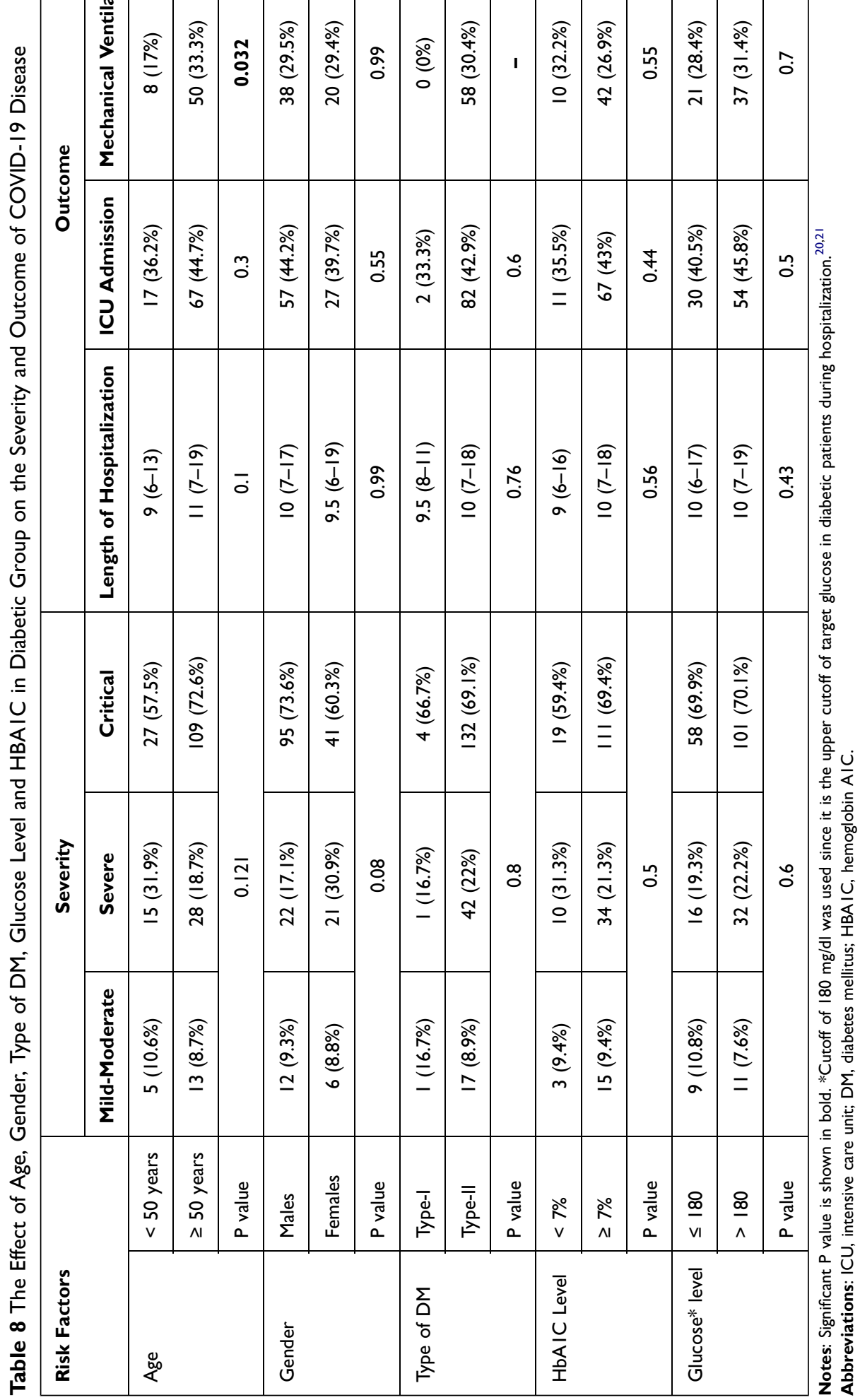


663 hospitalized COVID-19 patients who were admitted to a university hospital in Wuhan and found that $60 \%$ of their population had severe-critical disease. ${ }^{25}$

Our study showed that inflammatory and severityrelated biomarkers (WBCs, neutrophils, LDH, ESR, CRP and D-dimer) were higher in DM group (Table 3). In addition, BUN and creatinine indicating renal function were worse in DM group, meaning higher risk of acute kidney injury. Moreover, $\mathrm{CO} 2$, which is an indirect measure of bicarbonate level in the serum, was statistically lower in DM group, indicating higher risk of metabolic acidosis. These results are in line with a previous study of 52 diabetic patients with COVID-19 where patients with severe events had higher leucocyte, neutrophil counts, ESR, CRP, procalcitonin and D-dimer with lower lymphocyte count. In addition, the same study found that cardiac troponin I was an independent risk factor for severe events in diabetic patients. $^{26}$ Moreover, acute kidney injury was reported to be a complication of critical COVID-19 by Gupta et al. ${ }^{27}$ This supports our finding of worse kidney function in the DM group, which can be explained by their severe-critical presentation, as we will discuss further in the paper.

The majority of our diabetic patients presented with severe-critical disease in $21.8 \%$ and $69 \%$, respectively $(\mathrm{P}<0.0001)$. We also found that $51.5 \%$ of severe and $57.4 \%$ of critical cases had DM $(\mathrm{P}<0.0001)$. In addition, our diabetic group had higher occurrence of ARDS, sepsis, altered level of consciousness, multi-organ failure, cytokine storm syndrome $(\mathrm{P}<0.05)$. Moreover, multivariate analysis showed that DM is an independent risk factor for both severe and critical COVID-19 disease. Our findings correlate with previous evidence that found an association of DM with a higher risk of severe pneumonia in 16$40 \%{ }^{26,28}$ They were also found in the literature to have a greater occurrence of ARDS, acute heart injury, acute kidney injury, septic shock, and disseminated intravascular coagulation. ${ }^{11}$ McGurnagha et al reported that DM was associated with an OR of 1.395 for fatal or critical care unit treated COVID-19. ${ }^{29}$ Similar evidence was reported in a meta-analysis of 16,000 patients, where DM was found to be associated with severe COVID-19 with OR of $2.75 .^{30}$

Our study shows that DM is associated with a worse outcome (Table 5). Multivariate analysis confirmed that $\mathrm{DM}$ is an independent risk factor for ICU admission and death (Table 6). Previous evidence showed that DM was associated with $5.0 \%$ risk of ICU admission, $2.3 \%$ risk of invasive mechanical ventilation and $1.4 \%$ risk of death. ${ }^{28}$ In a study of 123 diabetic patients with COVID-19 infection, patients with DM required more non-invasive and invasive mechanical ventilation compared to patients without diabetes. ${ }^{31}$ Bode et al reported a longer median length of stay in 184 patients with DM and/or uncontrolled hyperglycemia compared to 386 patients without diabetes or hyperglycemia. ${ }^{23}$ Previous studies reported a mortality rate of around $17-20 \%$ in diabetic patients with COVID19 infection $22,24,31,32$ which is similar to our finding of $20 \%$ mortality rate. In the same study by Bode et al, DM was found to be associated with more than fourfold increase in mortality. ${ }^{23}$ Comparing those numbers with the reported outcome of COVID-19 infection in the general population, ${ }^{9,10}$ it becomes obvious that DM is associated with worse outcomes and mortality.

Interestingly, we found that hyperglycemia without pre-existing diabetes is associated with a higher risk of critical presentation in 75\% (P 0.001) with worse outcome in terms of longer hospitalization (P 0.014), higher risk of ICU admission, mechanical ventilation, and death $(\mathrm{P}<$ 0.0001). Similarly, Cai et al looked at a hyperglycemic group of patients without pre-existing DM and reported that hyperglycemia is associated with a higher risk of ARDS and acute respiratory failure. ${ }^{31}$ In addition, other complications like acute kidney injury, acute heart failure, electrolyte disturbance and hypoproteinemia were higher among hyperglycemic group. ${ }^{27}$ Zhang et al reported an association of hyperglycemia without DM with a higher risk of critical care admission, mechanical ventilation, and death. ${ }^{33}$ Moreover, mortality rate of COVID-19 was reported to be worse in patients with higher fasting blood glucose even when it is in the normal range (P 0.003). ${ }^{33}$

Multiple mechanisms were postulated to explain the worst outcome in diabetic or hyperglycemic patients with COVID-19 infection. First, high markers of inflammation such as CRP and D-dimer are strongly associated with critical COVID-19 infection and mortality. ${ }^{34}$ Diabetic patients were found to have higher levels of these markers ${ }^{26}$ indicating their higher risk of critical presentation and worse outcome. Second, COVID-19 disease has been associated with risk of thrombosis and abnormal coagulation pattern. $^{35}$ Non-survivors have significantly higher D-dimer, ${ }^{33,35}$ fibrin degradation product levels, longer prothrombin and activated partial thromboplastin time. ${ }^{35} \mathrm{DM}$ causes thrombosis since it predisposes to prothrombotic state secondary to endothelial dysfunction, coagulative activation and platelet hyperreactivity 
mediated by hyperglycemia, insulin resistance, inflammation and oxidative stress. ${ }^{36}$ Next, SARS-CoV-2 can cause $\beta$ cell damage which could lead to a new onset diabetes or sustained in-hospital hyperglycemia. ${ }^{37}$ In addition, it could precipitate acute hyperglycemic crisis in the form of diabetic ketoacidosis or hyperglycemic hyperosmolar state. ${ }^{37}$ Lastly, obesity is a very common comorbidity with DM. ${ }^{38}$ Many reports have shown a more severe COVID-19 illness and death in association with obesity even at young age. $^{39}$

Studying the effect of possible risk factors in DM group on the severity and outcome of COVID-19 infection, we found that only male gender tended to have a higher risk of critical presentation and older age $\geq 50$ years was associated with a higher risk of mechanical ventilation. However, neither the type of diabetes nor the level of control reflected by BG or HBA1C affected the severity or outcome of COVID-19 disease. However, we only had 7 cases of T1DM patients, which makes the conclusion regarding type of diabetes difficult in our study. In a multicentre study of 950 diabetic patients, the well-controlled glucose group had lower in-hospital complications and mortality as compared to individuals with poorly controlled BG level. ${ }^{11}$ However, in another study of 117 patients with T2DM and COVID-19, clinical outcomes and chest $\mathrm{CT}$ severity scores were similar between patients with well controlled and poorly controlled DM. ${ }^{32}$ Furthermore, a large comparative study showed that even diabetic patients with optimal control had an elevated risk of serious infection as compared to patients without diabetes, and the risks rose with increasing HBA1C and with T1DM especially with poor control. ${ }^{40}$ Therefore, most of the evidence supports our findings that having diabetes or hyperglycemia at presentation even without history of DM are considered by themselves risk factor for worse outcome regardless of the control of diabetes. However, some studies showed that patients with poor control did worse than wellcontrolled ones, which was not found in our study.

Looking into other risk factors of severe COVID-19 disease, our multivariate analysis showed that increasing age was an independent risk factor for severe COVID-19 disease, worse outcome, and mortality. Male gender was associated with critical disease, ICU admission and mechanical ventilation. CKD was associated only with critical presentation, and GI diseases were associated with worse outcome. Previously published data similarly showed that patients with severe pneumonia were older in age. ${ }^{4}$ Moreover, male patients were reported to have almost three times the odds of requiring intensive treatment unit admission and higher odds of death compared to females in a recent meta-analysis. ${ }^{41}$ Our findings are also comparable to previous evidence regarding CKD. In a meta-analysis of 4350 patients, CKD patients had a significantly increased risk of severe disease as compared to non-CKD patients with OR of $2.15 .^{42}$

\section{Conclusion}

Diabetes Mellitus is an independent risk factor for severe-critical COVID-19 disease, ICU admission and mortality. In addition, hyperglycaemia on presentation, even in the absence of pre-existing DM, worsens the severity and outcome of COVID-19 infection. Therefore, glucose testing, and glycemic control are crucial for all COVID-19 patients, even those without preexisting DM. Further studies to understand the exact pathogenesis of such findings are required to improve diabetic patients' outcome. The COVID-19 vaccination program should target such patients to prevent severe disease and lower mortality.

\section{Abbreviations}

COVID-19, Corona virus disease-2019; DM, Diabetes mellitus; ICU, intensive care unit; T2DM, Type 2 diabetes mellitus; HR, Hazard ratio; KSA, Kingdom of Saudi Arabia; LDH, Lactate dehydrogenase; ESR, Erythrocyte sedimentation rate; CRP, C-reactive protein; $\mathrm{MOH}$, Ministry of health; ARDS, Adult respiratory distress syndrome; HBA1C, glycosylated hemoglobin; BG, Blood glucose; IQR, Interquartile range; ORs, odds ratios; CI, Confidence intervals; CKD, Chronic kidney disease; GI, Gastrointestinal; T1DM, Type 1 diabetes mellitus; WBCs, White blood cell count; BUN, Blood urea nitrogen; K, Potassium; Na, Sodium; $\mathrm{Cl}$, Chloride; $\mathrm{CO} 2$, Carbon dioxide.

\section{Ethical Approval}

The study was approved by the Institutional Review Board (IRB) of Imam Abdulrahman Bin Faisal University with an approval number of (IRB-2020-01-248). Patients' consent to review their medical records was not required by the IRB of Imam Abdulrahman Bin Faisal University since it is a retrospective study. The data confidentiality and compliance with the Declaration of Helsinki were maintained.

\section{Source of Funding}

None declared. 


\section{Author Contributions}

All authors made a significant contribution to the work reported, whether that is in the conception, study design, execution, acquisition of data, analysis and interpretation, took part in drafting, revising and critically reviewing the article; gave final approval of the version to be published; have agreed on the journal to which the article has been submitted; and agree to be accountable for all aspects of the work.

\section{Disclosure}

The authors report no conflicts of interest in this work.

\section{References}

1. COVID-19 Coronavirus - update. Available from: https://virusncov. com/. Accessed October 29, 2021.

2. World Health organization. Saudi Arabia: WHO Coronavirus Disease (COVID-19) Dashboard. Available from: https://covid19.who.int/ region/emro/country/sa. Accessed June 6, 2021.

3. Wu Z, McGoogan J. Characteristics of and Important Lessons from the Coronavirus Disease 2019 (COVID-19) Outbreak in China Summary of a Report of 72314 Cases from the Chinese Center for Disease Control and Prevention. JAMA. 2020;323(13):1239-1242. doi:10.1001/jama.2020.2648

4. Lim S, Bae JH, Kwon HS, Nauck MA. COVID-19 and diabetes mellitus: from pathophysiology to clinical management. Nat Rev Endocrinol. 2021;17:11-30. doi:10.1038/s41574-020-00435-4

5. Pearson-Stuttard J, Blundell S, Harris T, Cook DG, Critchley J. Diabetes and infection: assessing the association with glycaemic control in population-based studies. Lancet Diabetes Endocrinol. 2016;4(2):148-158. doi:10.1016/S2213-8587(15)00379-4

6. Codo AC, Davanzo GG, Monteiro LB, et al. Elevated Glucose Levels Favor SARS-CoV-2 Infection and Monocyte Response through a HIF-1 $\alpha /$ Glycolysis-Dependent Axis. Cell Metab. 2020;32(3):437446.e5. doi:10.1016/j.cmet.2020.07.007

7. Zhou F, Yu T, Du R, et al. Clinical course and risk factors for mortality of adult inpatients with COVID-19 in Wuhan, China: a retrospective cohort study. Lancet. 2020;395(10229):1054-1062. doi:10.1016/S0140-6736(20)30566-3

8. McMichael T, Currie D, Clark S, et al. Epidemiology of Covid-19 in a Long-Term Care Facility in King County, Washington. $N$ Engl J Med. 2020;382:2005-2011. doi:10.1056/NEJMoa2005412

9. Li X, Xu S, Yu M, et al. Risk factors for severity and mortality in adult COVID-19 inpatients in Wuhan. $J$ Allergy Clin Immunol. 2020;146(1):110-118. doi:10.1016/j.jaci.2020.04.006

10. Du Y, Tu L, Zhu P, et al. Clinical Features of 85 Fatal Cases of COVID-19 from Wuhan. A Retrospective Observational Study. Am J Respir Crit Care Med. 2020;201(11):1372-1379. doi:10.1164/ rccm.202003-0543OC

11. Zhu L, She ZG, Cheng X, et al. Association of Blood Glucose Control and Outcomes in Patients with COVID-19 and Pre-existing Type 2 Diabetes. Cell Metab. 2020;31(6):1068-1077. doi:10.1016/j. cmet.2020.04.021

12. Roncon L, Zuin M, Rigatelli G, Zuliani G. Diabetic patients with COVID-19 infection are at higher risk of ICU admission and poor short-term outcome. J Clin Virol. 2020;127:104354. doi:10.1016/j. jcv.2020.104354
13. Aleanizy FS, Alqahtani FY, Alanazi MS, et al. Clinical characteristics and risk factors of patients with severe COVID-19 in Riyadh, Saudi Arabia: a retrospective study. J Infect Public Health. 2021;14 (9):1133-1138. doi:10.1016/j.jiph.2021.07.014

14. Iroungou BA, Mangouka LG, Bivigou-Mboumba B, et al. Demographic and Clinical Characteristics Associated With Severity, Clinical Outcomes, and Mortality of COVID-19 Infection in Gabon. JAMA Netw Open. 2021;4(9):e2124190. doi:10.1001/jamanetworkopen.2021.24190

15. Patone M, Thomas K, Hatch R, et al. Mortality and critical care unit admission associated with the SARS-CoV-2 lineage B.1.1.7 in England: an observational cohort study. Lancet Infect Dis. 2021;21:1518-1528. doi:10.1016/S1473-3099(21)00318-2

16. Meo SA. Prevalence and future prediction of type 2 diabetes mellitus in the Kingdom of Saudi Arabia: a systematic review of published studies. J Pak Med Assoc. 2016;66(6):722-725.

17. Villar J, Ariff S, Gunier RB, et al. Maternal and Neonatal Morbidity and Mortality Among Pregnant Women with and Without COVID-19 Infection: the INTERCOVID Multinational Cohort Study. JAMA Pediatr. 2021;175(8):817-826. doi:10.1001/jamapediatrics.2021.1050

18. Qeadan F, Mensah NA, Tingey B, et al. The risk of clinical complications and death among pregnant women with COVID-19 in the Cerner COVID-19 cohort: a retrospective analysis. BMC Pregnancy Childbirth. 2021;21:305. doi:10.1186/s12884-02103772-y

19. Saudi Arabian Ministry of Health. Saudi MOH Protocol for Patients Suspected of/Confirmed with COVID-19. Supportive care and antiviral treatment of suspected or confirmed COVID-19 infection. (Version 2.9) May 19th, 2021. Available from: https://www.moh. gov.sa/Ministry/MediaCenter/Publications/Documents/MOHtherapeutic-protocol-for-COVID-19.pdf. Accessed June 3, 2021.

20. Umpierrez GE, Hellman R, Korytkowski MT, et al. Management of hyperglycemia in hospitalized patients in non-critical care setting: an endocrine society clinical practice guideline. $J$ Clin Endocrinol Metab. 2012;97(1):16-38. doi:10.1210/jc.2011-2098

21. American diabetes Association. Diabetes Care in the Hospital: standards of medical Care in Diabetes-2021. Diabetes Care. 2021;44 (Suppl 1):S211-S220. doi:10.2337/dc21-S015

22. Alguwaihes AM, Al-Sofiani ME, Megdad M, et al. Diabetes and Covid-19 among hospitalized patients in Saudi Arabia: a single-centre retrospective study. Cardiovasc Diabetol. 2020;19 (1):205. doi:10.1186/s12933-020-01184-4

23. Bode B, Garrett V, Messler J, et al. Glycemic Characteristics and Clinical Outcomes of COVID-19 Patients Hospitalized in the United States. J Diabetes Sci Technol. 2020;14(4):813-821. doi:10.1177/ 1932296820924469

24. Ciceri F, Castagna A, Rovere-Querini P, et al. Early predictors of clinical outcomes of COVID-19 outbreak in Milan, Italy. Clin Immunol. 2020;217:108509. doi:10.1016/j.clim.2020.108509

25. Wang X, Liu Z, Li J, et al. Impacts of Type 2 Diabetes on Disease Severity, Therapeutic Effect, and Mortality of Patients With COVID-19. J Clin Endocrinol Metab. 2020;105(12):e4219-e4229. doi:10.1210/clinem/dgaa535

26. Zhang N, Wang C, Zhu F, et al. Risk Factors for Poor Outcomes of Diabetes Patients With COVID-19: a Single-Center, Retrospective Study in Early Outbreak in China. Front Endocrinol. 2020;24 (11):571037. doi:10.3389/fendo.2020.571037

27. Gupta S, Hayek SS, Wang W, et al. Factors Associated With Death in Critically Ill Patients With Coronavirus Disease 2019 in the US [published correction appears in JAMA Intern Med. JAMA Intern Med. 2020;180(11):1436-1447. doi:10.1001/jamainternmed.2020.3596

28. Guan W, Ni Z, Hu Y, et al. Clinical characteristics of coronavirus disease 2019 in China. $N$ Engl J Med. 2020;382:1708-1720. doi:10.1056/NEJMoa2002032 
29. McGurnaghan SJ, Weir A, Bishop J, et al. Risks of and risk factors for COVID-19 disease in people with diabetes: a cohort study of the total population of Scotland. Lancet Diabetes Endocrinol. 2021;9 (2):82-93. doi:10.1016/S2213-8587(20)30405-8

30. Kumar A, Arora A, Sharma P, et al. Is diabetes mellitus associated with mortality and severity of COVID-19? A meta-analysis. Diabetes Metab Syndr. 2020;14(4):535-545. doi:10.1016/j.dsx.2020.04.044

31. Cai Y, Shi S, Yang F, et al. Fasting blood glucose level is a predictor of mortality in patients with COVID-19 independent of diabetes history. Diabetes Res Clin Pract. 2020;169:108437. doi:10.1016/j. diabres.2020.108437

32. Raoufi M, Khalili S, Mansouri M, Mahdavi A, Khalili N. Wellcontrolled vs poorly-controlled diabetes in patients with COVID-19: are there any differences in outcomes and imaging findings? Diabetes Res Clin Pract. 2020;166:108286. doi:10.1016/j.diabres.2020.108286

33. Zhang Y, Li H, Zhang J, et al. The clinical characteristics and outcomes of patients with diabetes and secondary hyperglycaemia with coronavirus disease 2019: a single-centre, retrospective, observational study in Wuhan. Diabetes Obes Metab. 2020;22 (8):1443-1454. doi:10.1111/dom.14086

34. Petrilli CM, Jones SA, Yang J, et al. Factors associated with hospital admission and critical illness among 5279 people with coronavirus disease 2019 in New York City: prospective cohort study. BMJ. 2020;369:m1966. doi:10.1136/bmj.m1966

35. Tang N, Li D, Wang X, Sun Z. Abnormal coagulation parameters are associated with poor prognosis in patients with novel coronavirus pneumonia. J Thromb Haemost. 2020;18(4):844-847. doi:10.1111/ jth. 14768
36. Natale V, Paola R, Chiara C, Giovanni D. Diabetes mellitus and thrombosis. Thromb Res. 2012;129(3):371-377. doi:10.1016/j. thromres.2011.11.052

37. Apicella M, Campopiano MC, Mantuano M, Mazoni L, Coppelli A, Del Prato S. COVID-19 in people with diabetes: understanding the reasons for worse outcomes. Lancet Diabetes Endocrinol. 2020;8 (9):782-792. doi:10.1016/S2213-8587(20)30238-2

38. Knowler WC, Pettitt DJ, Savage PJ, Bennett PH. Diabetes incidence in Pima Indians: contributions of obesity and parental diabetes. Am J Epidemiol. 1981;113(2):144-156. doi:10.1093/oxfordjournals.aje. a113079

39. Hendren NS, de Lemos JA, Ayers C, et al. Association of Body Mass Index and Age With Morbidity and Mortality in Patients Hospitalized With COVID-19: results From the American Heart Association COVID-19 Cardiovascular. Circulation. 2021;143(2):135-144. doi:10.1161/CIRCULATIONAHA.120.051936

40. Critchley JA, Carey IM, Harris T, deWilde S, Hosking FJ, Cook DG. Glycemic control and risk of infections among people with type 1 or type 2 diabetes in a large primary care cohort study. Diabetes Care. 2018;41:2127-2135. doi:10.2337/dc18-0287

41. Peckham H, de Gruijter NM, Raine C, et al. Male sex identified by global COVID-19 meta-analysis as a risk factor for death and ITU admission. Nat Commun. 2020;11:6317. doi:10.1038/s41467-02019741-6

42. Menon T, Gandhi SAQ, Tariq W, et al. Impact of Chronic Kidney Disease on Severity and Mortality in COVID-19 Patients: a Systematic Review and Meta-analysis. Cureus. 2021;13(4): e14279. doi:10.7759/cureus.14279
International Journal of General Medicine

\section{Publish your work in this journal}

The International Journal of General Medicine is an international, peer-reviewed open-access journal that focuses on general and internal medicine, pathogenesis, epidemiology, diagnosis, monitoring and treatment protocols. The journal is characterized by the rapid reporting of reviews, original research and clinical studies

\section{Dovepress}

across all disease areas. The manuscript management system is completely online and includes a very quick and fair peer-review system, which is all easy to use. Visit http://www.dovepress.com/ testimonials.php to read real quotes from published authors. 